\title{
Exérese de lipoma gigante na Clínica da Família: um relato de caso
}

\author{
Giant lipoma excision in the Family Clinic: a case report \\ Escisión de lipoma gigante en la Clínica Familiar: reporte de un caso
}

Roberto Wallace Dias Lacerda ${ }^{a}$, Cássio Battisti Serafinib, Pedro Miño Viannac ${ }^{c}$, Leandro Jácomo Mariab ${ }^{b}$ Giulia Garcia Regattieric

\section{Resumo}

A realização de procedimentos cirúrgicos na Unidade Básica de Saúde é preconizada e descrita na literatura. No entanto, tal prática vem sendo cada vez menos aplicada na atenção primária. Em um contexto de superlotações de hospitais terciários e consequente inoperação de cirurgias eletivas, é de extrema importância a realização desses procedimentos na Clínica da Família. Nesse trabalho, apresenta-se um caso de um paciente submetido à exérese de lipoma gigante incapacitante, na fossa poplítea esquerda, com 12 anos de evolução e com tentativa frustrada de resolução cirúrgica em um hospital terciário.

\section{Abstract}

The implementation of surgical procedures in the Basic Health Unit is recommended and described in the literature. However, such practice has been less and less applied in primary care. In a context of overcrowding of tertiary hospitals and consequent inoperability of elective surgical procedures it is of utmost importance and resolutivity carrying out these procedures in the Family Clinic/Basic Health Unit. In this article, we present a case of a patient who underwent excision of a crippling giant lipoma in the left popliteal fossa, with 12 years of evolution and unsuccessful attempts of surgical resolution in a tertiary hospital.

\section{Resumen}

Los procedimientos quirúrgicos en la Unidad Básica de Salud se recomiendan y se describen en la literatura. Sin embargo, esta práctica se está convirtiendo en una menor aplicación en atención primaria. En un contexto de hacinamiento en hospitales de tercer nivel de atención y dando como resultado la inoperabilidad de las quirugías menores electivas, es extremadamente importante realizar estos procedimientos en la Clínica de la Familia/Unidad Basica de Salud. En este trabajo, se presenta un caso de un paciente que se submetió a la extirpación de incapacitante lipoma gigante en la fosa poplítea izquierda con 12 años de evolución y frustra resolución quirúrgica en un hospital de tercer nivel.
Palavras-chave:

Lipoma

Procedimentos Cirúrgicos

Ambulatoriais

Integralidade em Saúde

Sistema Único de Saúde

Estratégia Saúde da Família

\section{Keywords:}

Lipoma

Ambulatory Surgical

Procedures

Integrality in Health

Unified Health System

Family Health Strategy

\section{Palabras clave:}

Lipoma

Procedimientos Quirúrgicos

Ambulatorios

Integralidad em Salud

Sistema Único de Salud

Estrategia de Salud Familiar
Como citar: Lacerda RWD, Serafini CB, Vianna PM, Maria LJ, Regattieri GG. Exérese de lipoma gigante na Clínica da Família: um relato de caso. Rev Bras Med Fam Comunidade. 2017;12(39):1-10. http://dx.doi.org/10.5712/rbmfc12(39)1294
Fonte de financiamento: declaram não haver.

Parecer CEP:

não se aplica.

Conflito de interesses:

declaram não haver.

Procedência e revisão por pares: revisado por pares.

Recebido em: 01/04/2016.

Aprovado em: 27/12/2016. 


\section{Introdução}

O SUS foi criado em 1988 com a proposta de reorientar o modelo de saúde vigente no país, seguindo os preceitos de Universalidade, Integralidade e Equidade. Na Lei 8080, de 1990, que organizou os princípios do SUS, além das três características já citadas, incluíram-se hierarquização, complementariedade, participação popular e resolubilidade aos atributos desse sistema público. ${ }^{1,2}$ É nesse contexto que a especialização em Medicina de Família e Comunidade (MFC) traz como habilidades do médico de família a realização de pequenos procedimentos cirúrgicos, na própria unidade de saúde. Tais atributos também são preconizados em outros países, nos quais a Medicina de Família é bem estruturada. ${ }^{3}$

Entende-se que a Estratégia Saúde da Família (ESF) deve ser porta de entrada para o Sistema, oferecendo atendimento longitudinal à população territorialmente adscrita, com criação de vínculo, e ter abordagem Integral ao paciente. Pode-se perceber o atributo de Resolubilidade do SUS dentro da ESF quando se diz que esta trabalha com elevada complexidade e baixa densidade. ${ }^{4}$

Na Clínica da Família Zilda Arns (CFZA), localizada no Complexo do Alemão - Rio de Janeiro (Brasil), desde maio de 2015 se tem acumulado experiência com pequenos procedimentos cirúrgicos, no âmbito de assistência à população, treinamento de médicos residentes em MFC e ensino a graduandos de universidades conveniadas. Dentre os procedimentos já realizados, se destacam: drenagem de abscessos, suturas, cantoplastias, exérese de lipomas, cistos sebáceos, fibroma mole, nevos, retiradas de corpos estranhos, lobuloplastias, cauterizações de verrugas, vasectomias, paracenteses de alívio, toracocenteses diagnósticas e, infiltrações de corticóide intra-articular. No ano de 2016 foram realizadas, 107 lipomas simples, 5 postectomias, 5 excisões cirúrgicas de lipomas gigantes, dentre outros, totalizando cerca de 400 procedimentos cirúrgicos.

Dentre as patologias cirúrgicas realizadas na CFZA, o lipoma se mostrou prevalente na casuística do referido período, representando 107 casos dos aproximados 400 procedimentos documentados. Sabe-se que o lipoma é definido com um tumor benigno de células adiposas maduras, sendo o tumor benigno mesenquimal de tecidos moles mais frequente na população. Sua prevalência é maior em indivíduos entre 40 a 60 anos de idade e pode estar presente em qualquer área do corpo em que há tecido adiposo (pescoço, dorso, nádegas, extremidades, bem como visceral - apresentação incomum). ${ }^{5-7}$

Possui crescimento lento e insidioso, sem invasão local e, na maioria das vezes, é assintomático. Uma apresentação clínica comum é um nódulo regular ou bocelado, subcutâneo, extremamente móvel e indolor. No entanto, quando apresenta grandes volumes, pode evoluir com dor local e compressão de estruturas vasculo-nervosas adjacentes, gerando sintomatologia mais exuberante como dor neuropática, parestesias, paresia, trombose venosa. Para ser caraterizado como lipoma gigante, deve apresentar mais de $10 \mathrm{~cm}$ ou mais de $1000 \mathrm{~g} .{ }^{8}$

O diagnóstico dos lipomas inicialmente é clínico, porém, exames complementares de imagem são úteis para excluir malignização. Ultrassonografia de partes moles ou ressonância magnética podem revelar septos espessos, áreas nodulares não adiposas ou redução do percentual de tecido gorduroso em sua composição, denotando malignidade. ${ }^{9}$

O tratamento de escolha consiste na exérese cirúrgica, para alívio de sintomas, bem como para diagnóstico definitivo histopatológico. ${ }^{10} \mathrm{~A}$ lipoaspiração é uma alternativa descrita na literatura, porém sua indicação em lipomas gigantes é controversa, devido ao seu risco de malignização. 
Os pacientes devem ser seguidos a longo prazo, visto que existe tendência à recidiva dessas lesões. Objetivo

O presente trabalho é um relato de caso de exérese de lipoma gigante realizado em uma Clínica da Família da Zona Norte do Rio de Janeiro, com a participação de preceptores da Residência de Medicina de Família e Comunidade do Município do Rio de Janeiro (RMFC-Rio), residentes em Medicina de Família, e acadêmicos de Medicina em Estágio supervisionado.

Pretende-se relatar a experiência dos Médicos de Família desta unidade e trazer a público a discussão sobre a realização de procedimentos cirúrgicos ambulatoriais na Estratégia Saúde da Família, sua aplicabilidade prática e o impacto sobre o sistema de saúde.

\section{Detalhamento do caso}

Paciente masculino, 65 anos, natural do Pará (Brasil), residente do Rio de Janeiro, profissional autônomo, mora com a esposa e 3 de seus 8 filhos. Hipertenso e diabético em uso de ácido acetilsalicílico, anlodipino, atenolol, losartana, metformina e glibenclamida. Apresentou acidente vascular encefálico isquêmico (AVEi) em 2009, evoluindo com hemiparesia à direita e disartria. Após três meses de tratamento fisioterápico, apresentou melhora importante, com atual força grau $V$ nos membros superior e inferior esquerdos, e grau IV em membros superior e inferior direitos, e disartria residual. Negava cirurgias prévias, alergias ou transfusões sanguíneas.

Ao exame físico, apresentava massa regular fibroelástica em subcutâneo de região poplítea esquerda, de aproximadamente $17 \mathrm{~cm}$, ligeiramente móvel, dolorosa à palpação e durante a marcha (Figura 1). Relatava que essa massa foi percebida há 12 anos (2003) e apresentou crescimento lento durante todo o período. O paciente deambulava com auxílio de muletas e se encontrava com o humor deprimido pois, segundo o mesmo, teve que abandonar o trabalho de pintor, já que sua perna não acometida pelo AVEi agora apresentava uma tumoração dolorosa, que o incapacitava para sua atividade laboral, além de prejuízo estético.

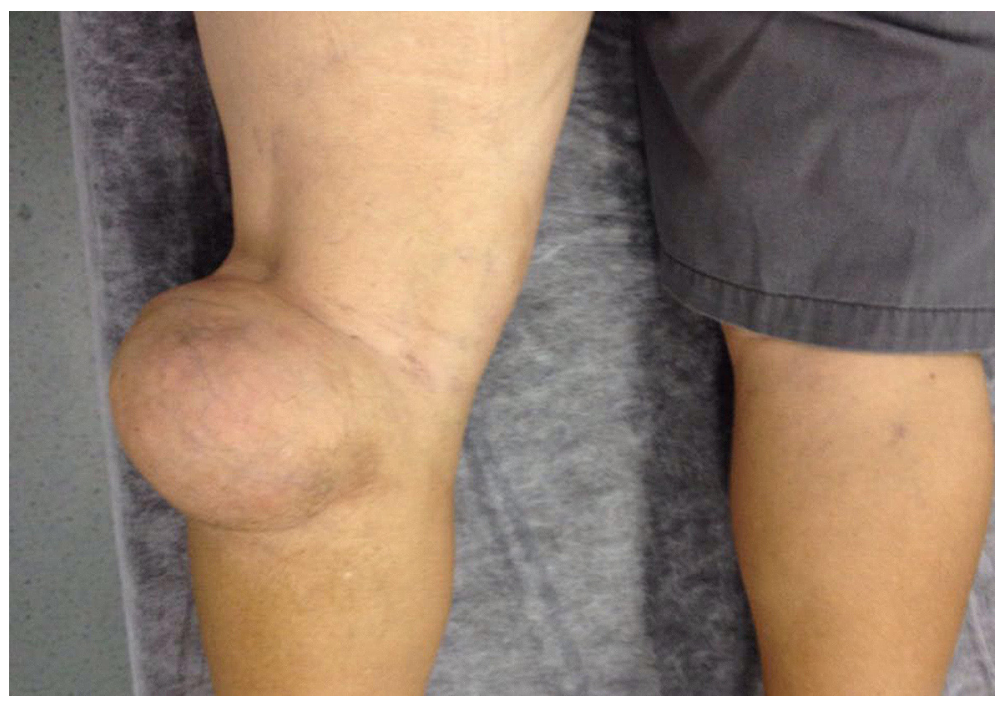

Figura 1. Lipoma gigante na região poplítea esquerda. 
Relatava também internação prévia em um Hospital Geral (terciário) da região para ser submetido à exérese da lesão, no entanto, após duas semanas de investigação diagnóstica e diversas remarcações de sua cirurgia, foi concedida alta hospitalar sem o tratamento definitivo de sua patologia.

Foi proposto o tratamento cirúrgico na própria Clínica da Família. Para isso, foi realizado ultrassonografia de partes moles que revelou tecido celular subcutâneo com nódulo ecogênico com linhas e calcificações de permeio, contorno irregular, medindo aproximadamente $178 \times 90 \mathrm{~mm}$, sugestivo de lipoma.

Foi então agendada biópsia incisional/excisional, a depender dos achados do peroperatório.

Após assepsia/antissepsia com clorexidina degermante e alcoólica, foi realizada a anestesia local perilesional (infiltração do anestésico nas margens laterais e inferior da lesão) com cloridrato de lidocaína $2 \%$ com vasoconstrictor, foram colocados campos estéreis e realizada incisão transversal seguindo as linhas de tensão muscular da região da panturrilha esquerda (Figura 2). Após dissecção por planos, foi encontrada a massa com plano de clivagem em todas as suas bordas (Figura 3).

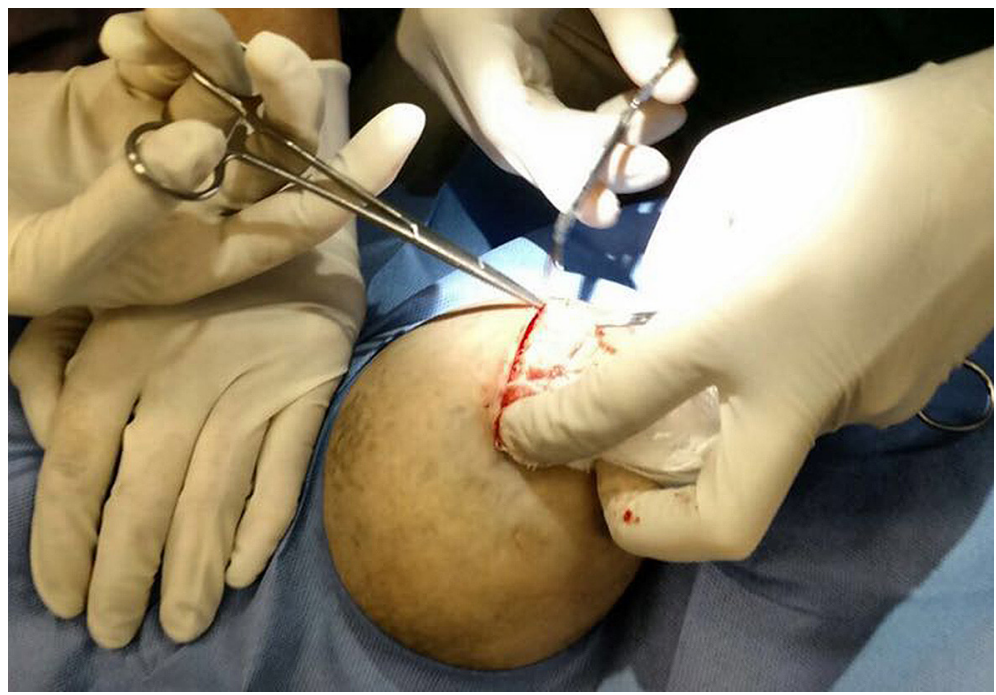

Figura 2. Incisão transversal seguindo as linhas de tensão muscular da região da panturrilha esquerda.

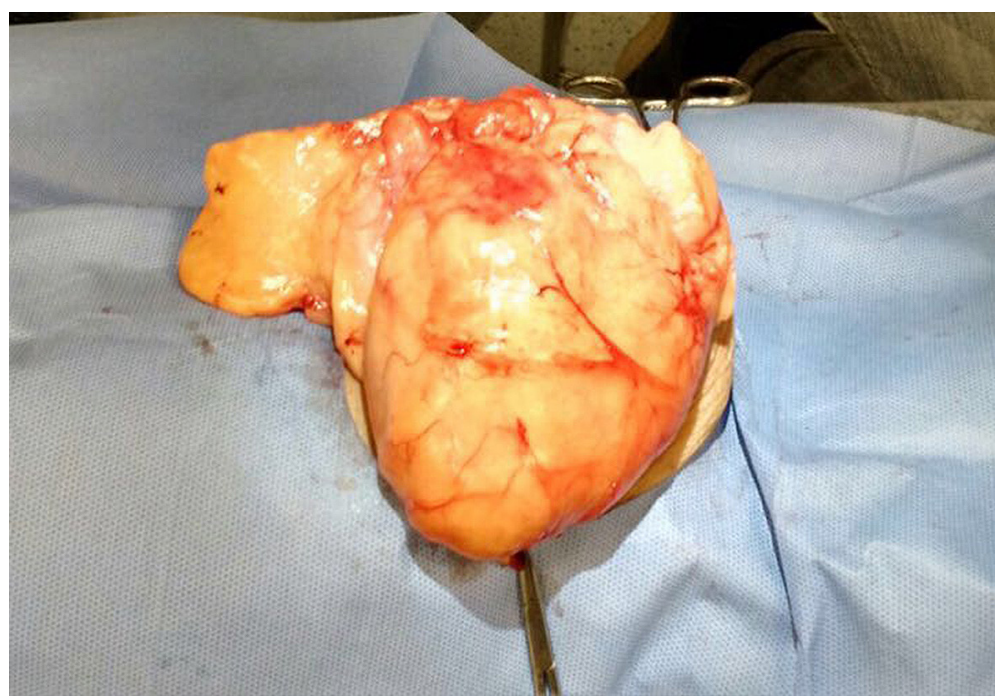

Figura 3. Exposição da massa tumoral. 
Devido à facilidade técnica e aspecto macroscópico sugestivo de benignidade (ausência de áreas de degeneração, necrose, focos de sangramento importantes ou pedículo vascular próprio), optou-se pela realização de exérese total da massa. O procedimento durou aproximadamente duas horas.

Após a enucleação do tumor foi realizada revisão hemostática com eletrocoagulaçao e ligadura de vasos dissecados com Categut 4.0. Foi realizado fechamento por planos com fio Nylon 3.0, com ponto hemostático de Donatti e fixação de dreno de Penrose na loja tumoral. Realizado curativo compressivo com gase e compressas estéreis, prescrito ibuprofeno VO por 5 dias e gelo tópico.

A peça cirúrgica apresentava 17,5 cm e 650 gramas, lobulada e homogênea, com aspecto de tecido adiposo e foi encaminhada para análise histopatológica após fragmentação em três peças, para facilitar transporte e armazenamento.

Laudo histopatológico revelo-se compatível com lipoma gigante:

Macroscopia: Três fragmentos de tecido brancacento-amarelado, homogêneo, lobulado e elástico, de, respectivamente, $5,5 \mathrm{~cm} \times 4,5 \mathrm{~cm} ; 5,5 \times 4 \mathrm{~cm} ; 6,5 \times 6,5 \mathrm{~cm}$, sem áreas de endurecimento ou degeneração aparente.

Microscopia: Tecido adiposo maduro típico entremeado por traves de tecido conjuntivo e capilares de permeio nas três peças analisadas. Sem sinais de degeneração sarcomatosa, esteatonecrose ou infiltrado inflamatório.

O dreno foi retirado com 48 horas de pós-operatório (Figura 4). O paciente não apresentou febre ou demais sinais de infecção de sítio cirúrgico, sendo acompanhado com retornos a cada três dias na própria clínica, com progressiva melhora. As suturas foram retiradas com 15 dias de pós-operatório.

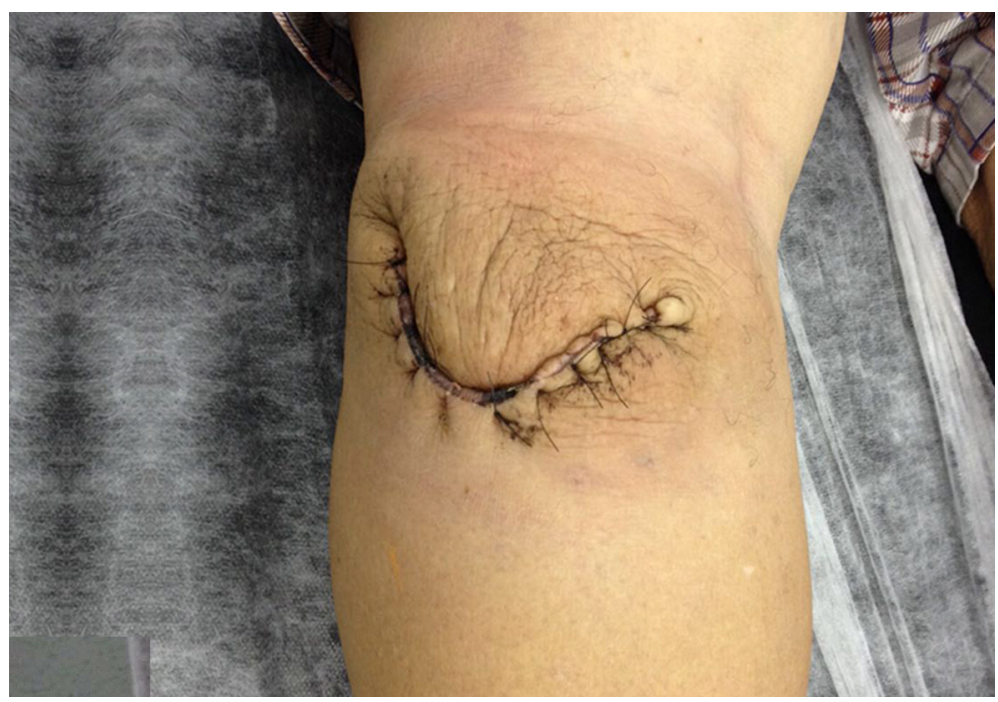

Figura 4. Ferida operatória após retirada do dreno - 48 horas após o procedimento.

O paciente se mostrou satisfeito com o resultado nas consultas de revisão, com ganhos em sua aparência, autoconfiança, autonomia nas atividades diárias e, inclusive, retorno às suas atividades laborais. Relatou não depender mais de muletas para deambular ter melhora substancial na qualidade de vida. 
Materiais necessários para o procedimento:

1. Clorexidina degermante - 1 frasco

2. Clorexidina alcoólica - 1 frasco

3. Cloridrato de lidocaína $2 \%$ com vasoconstrictor -2 frascos

4. Soro fisiológico $0,9 \%-500 \mathrm{ml}$

5. Campo estéril fenestrado

6. EPI - Máscara, touca, óculos, capote e luva estéril - 1 por integrante da equipe

7. Compressa de gaze estéril - 10 pacotes

8. Kit cirúrgico completo:

- Cabo de bisturi n.3;

- Lamina de bisturi n.11;

- Pinça Adson 14cm 1x2 dentes - 2 unidades;

- Pinça Halstead mosquito 12cm curva - 2 unidades;

- Pinça Kelly curva $14 \mathrm{~cm}-4$ unidades;

- Porta agulha Mayo Hegar 14cm;

- Tesoura Mayo Stille reta $15 \mathrm{~cm}$;

- Farabeuf;

- Metzenbaum $16 \mathrm{~cm}$.

9. Fio de sutura Catgut $4.0-2$ unidades

10. Fio de sutura Nylon 3.0 - 3 unidades

11. Dreno de Penrose - 1 unidade

\section{Descrição Cirúrgica}

Caso necessário e o paciente se mostre ansioso com o procedimento, administrar um comprimido de diazepam de $10 \mathrm{mg}$ com horas de antecedência, se não houver contraindicação. Neste relato não houve necessidade.

1. Assepsia;

2. Antissepsia;

3. Colocação de campo estéril fenestrado;

4. Aplicação de anestesia local em base lesional (8ml em quatro pontos diferentes);

5. Aplicação de anestesia local na linha de incisão;

6. Incisão fusiforme sobre a lesão de acordo com a linha de tensão;

7. Diérese do tecido lipomatoso do tecido subcutâneo tomando cuidado com possíveis vasos e hemostase imediata caso necessária;

8. Durante a diérese foram realizadas três hemostasias de pequenos vasos com catgut 4.0;

9. Exérese do tecido lipomatoso de forma circular;

10. Revisão hemostática;

11. Sutura com ponto em Donatti; 
12. Colocação de dreno de Penrose;

13. Compressão com gaze estéril e curativo com atadura;

14. Revisão diária nos primeiros 3 dias.

\section{Discussão}

O caso supracitado foi um entre os muitos exemplos de sucesso de procedimentos cirúrgicos realizados por Médicos de Família e comunidade capacitados, residentes e acadêmicos em formação sob supervisão na Clínica da Família Zilda Arns.

O paciente apresentava um lipoma gigante com 12 anos de evolução, que gerava importante impacto em sua vida, sob os pontos de vista orgânico, psíquico e social. Chegou a realizar exames pré-operatórios e foi internado em um hospital terciário para realizar a cirurgia, porém não teve sua doença tratada por gargalos administrativo-operacionais do Sistema Único de Saúde vigente.

Entende-se que a CF, por fazer parte da Estratégia Saúde da Família, deve ser resolutiva e, por isso, capaz de reduzir ao máximo o número de referenciamentos para especialistas ou unidades hospitalares. Ao realizar procedimentos como o descrito neste estudo, Médicos de Família se tornam capazes de desempenhar medicina de alta complexidade com a baixa densidade tecnológica, atuando de forma incisiva no manejo da integralidade do cuidado, aumentando sua autoconfiança e sendo cada vez mais atuantes no processo terapêutico de seus pacientes. . $^{3,11,12}$

A abrangência da atuação do médico de família no que se refere a procedimentos cirúrgicos ambulatoriais é muito ampla e varia desde os mais simples (drenagem de abscesso, retirada de corpo estranho nasal e auricular, cantoplastia) até os mais complexos e inusitados (toracocentese, paracentese, redução de luxações).$^{13}$ Essas técnicas requerem normalmente anestésico locais e apresentam poucos riscos e complicações. ${ }^{14,15}$

Na Clínica da Família em questão os procedimentos são realizados no período da tarde em três dias da semana (segundas, quartas e sextas-feiras), sendo os procedimentos mais complexos realizados às quartas-feiras. Foi criada uma agenda única de procedimentos, em que os médicos da família podem agendar os procedimentos cirúrgicos de acordo com a complexidade dos mesmos e com a disponibilidade de horários da sala de procedimentos.

Como se trata de uma Unidade Escola, com residência e internato, organizamos esses horários distribuídos ao longo da semana para que todos participem, porém é viável a sua replicação, já que reservamos não mais do que duas horas para estas atividades por dia, que poderia ser ajustado dentro de uma única equipe em um turno para procedimentos ou horários pequenos, distribuído na agenda de acordo com o procedimento realizado.

Na unidade, nos anos de 2015 e 2016, foram realizados aproximadamente 400 procedimentos cirúrgico-ambulatoriais/ano. Assim como o paciente discutido, outros tantos se beneficiaram com a realização dos procedimentos propostos no currículo baseado em competência da Sociedade Brasileira de Medicina de Família e Comunidade.

A capacitação desses médicos para procedimentos cirúrgicos ambulatoriais é preconizada e vantajosa por diversos motivos. ${ }^{16,17}$ Reduz os custos do sistema, já que essas enfermidades podem ser 
tratadas em fases iniciais, com menores complicações; ${ }^{11,18,19}$ reduz o tempo de espera dos pacientes pelo procedimento; ${ }^{20,21}$ e, a taxa de satisfação dos usuários é maior quando os procedimentos são realizados na Atenção Primária. ${ }^{3,11,12}$

A proporção de complicações cirúrgicas descrita na literatura é similar entre os procedimentos realizados por Médicos de Família e Especialistas; ${ }^{11,22}$ dessa forma, permite que patologias cirúrgicas

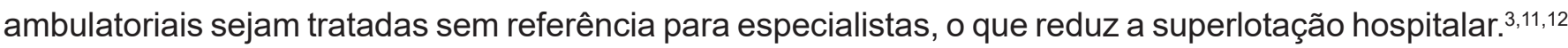
Na literatura é observada concordância entre o diagnóstico clínico e anatomopatológico. ${ }^{23-26}$

$\mathrm{Na}$ literatura é descrito que os Médicos de Família executam e ensinam mais procedimentos ambulatoriais em adultos do que os Médicos Internistas, por receberem mais treinamento e se sentirem mais confiantes em suas habilidades. ${ }^{16,17}$ Sua confiança esteve relacionada a executar ou participar de um mesmo procedimento pelo menos 10 vezes/ano. ${ }^{27}$

O avanço na realização de pequenos procedimentos cirúrgicos depende de mais Médicos de Família treinados, executando procedimentos cirúrgicos ambulatoriais e de novos relatos de casos e estudos demonstrando a aplicabilidade destas práticas com suas referidas taxas de sucesso. ${ }^{16,17,24} \mathrm{~A}$ autonomia dos Médicos de Família pode ser ampliada, além de beneficiar acadêmicos de Medicina que estagiam em ESF, que estão cada vez mais precocemente inseridos nesse contexto de aprendizagem.4,18

A partir do presente estudo, pode-se perceber que a realização de cirurgias de caráter ambulatorial em Clínicas da Família é factível. Para isso, porém, é necessário que haja estrutura física, material e treinamento/capacitação para que os Médicos de Família possam atuar da melhor maneira para o paciente e para o sistema de saúde como um todo.

Os atuais desafios para a diversificação dessa prática são bem documentados na literatura internacional, em que se destacam a falta de habilidade/treinamento atualizado e a falta de tempo. ${ }^{28} \mathrm{~A}$ aplicação de cursos práticos pode encorajar Médicos de Família a realizar pequenas cirurgias em suas clínicas e, consequentemente, ampliar o número de serviços realizados na atenção primária. ${ }^{29}$

\section{Referências}

1. Pinheiro R, Ceccim RB, Mattos RA, orgs. Ensino-Trabalho-Cidadania: novas marcas ao ensinar integralidade no SUS. Rio de Janeiro: IMS/UERJ/CEPESC/ABRASCO; 2006. 180 p.

2. Costa JRB, Romano VF, Costa RR, Vitorino RR, Alves LA, Gomes AP, et al. Formação médica na estratégia de saúde da família: percepções discentes. Rev Bras Educ Med. 2012;36(3):387-400.

3. Serra M, Arévalo A, Ortega C, Ripoll A, Giménez N. Minor surgery activity in primary care. JRSM Short Rep. 2010;1(4):36. DOI: http://dx.doi.org/10.1258/shorts.2009.090035

4. Ferreira RC, Silva RF, Aguer CB. Formação do Profissional Médico: a Aprendizagem na Atenção Básica de Saúde. Rev Bras Educ Med. 2007;31(1):52-59. DOI: http://dx.doi.org/10.1590/S0100-55022007000100008

5. Singh M, Saxena A, Kumar L, Karande SK, Kolhe Y. Giant lipoma of posterior cervical region. Case Rep Surg. 2014;2014:289383.

6. Brandler TI. Large fibrolipoma. Br Med J. 1894;1:574.

7.Terzioglu A, Tuncali D, Yuksel A, Bingul F, Aslan G. Giant lipomas: a series of 12 consecutive cases and a giant liposarcoma of the thigh. Dermatol Surg. 2004;30(3):463-7. DOI: http://dx.doi.org/10.1097/00042728-200403000-00033

8. Sanchez MR, Golomb FM, Moy JA, Potozkin JR. Giant lipoma: case report and review of the literature. J Am Acad Dermatol. 1993;28(2 Pt 1):266-8. DOI: http://dx.doi.org/10.1016/S0190-9622(08)81151-6 
9. Yakubu AA, Edino ST, Mohammed AZ, Sheshe AA, Alhassan SU. Giant and complicated subcutaneous lipoma of the neck. West Afr J Med. 2008;27(1):44-6.

10. Leuzzi G, Cesario A, Parisi AM, Granone P. Chest wall giant lipoma with a thirty-year history. Interact Cardiovasc Thorac Surg. 2012;15(2):323-4. DOI: http://dx.doi.org/10.1093/icvts/ivs159

11. George S, Pockney P, Primrose J, Smith H, Little P, Kinley H, et al. A prospective randomised comparison of minor surgery in primary and secondary care. The MiSTIC trial. Health Technol Assess. 2008;12(23):iii-iv, ix-38. DOI: http://dx.doi. org/10.3310/hta12230

12. Godfrey E, Watkiss M, Schnieden H. Initiation and evaluation of a pilot scheme for minor surgery in general practice. Health Trends. 1990;22(2):57-9.

13. Kelly BF, Sicilia JM, Forman S, Ellert W, Nothnagle M. Advanced procedural training in family medicine: a group consensus statement. Fam Med. 2009;41(6):398-404.

14. Friedlich M, MacRae H, Oandasan I, Tannenbaum D, Batty H, Reznick R, et al. Structured assessment of minor surgical skills (SAMSS) for family medicine residents. Acad Med. 2001;76(12):1241-6. DOI: http://dx.doi.org/10.1097/00001888200112000-00019

15. Menon NK. Minor surgery in general practice. Practitioner. 1986;230(1420):917-9.

16. Arroyo A, Andreu J, García P, Jover S, Arroyo M, Fernández A, et al. Analysis of a programme of direct referral between primary and specialist care in potential surgery patients. Aten Primaria. 2001;28(6):381-5.

17. Stainforth J, Goodfield MJ. Cost effectiveness of minor surgery in general practice. Br J Gen Pract. 1992;42(360):302-3.

18. Foulkes A. Minor surgery in general practice. BMJ. 1993;307(6905):685. DOI: http://dx.doi.org/10.1136/bmj.307.6905.685

19. Brown JS, Smith RR, Cantor T, Chesover D, Yearsley R. General practitioners as providers of minor surgery--a success story? Br J Gen Pract. 1997;47(417):205-10.

20. Pockney P, George S, Primrose J, Smith H, Kinley H, Little P, et al. Impact of the introduction of fee for service payments on types of minor surgical procedures undertaken by general practitioners: observational study. J Public Health (Oxf). 2004;26(3):264-7. DOI: http://dx.doi.org/10.1093/pubmed/fdh152

21. Tárraga López PJ, Marín Nieto E, García Olmo D, Celada Rodríguez A, Solera Albero J. Economic impact of the introduction of a minor surgery program in primary care. Aten Primaria. 2001;27(5):335-8. DOI: http://dx.doi.org/10.1016/S02126567(01)79377-8

22. Nunes PAL, Tambelli RA. A realização de pequenas cirurgias na Atenção Primária à Saúde. In: Anais do 120 Congresso Brasileiro de Medicina de Família e Comunidade. 201329 Maio 29-Jun 2; Belém, PA, Brasil. p. 268.

23. Landis JR, Koch GG. The measurement of observer agreement for categorical data. Biometrics. 1977;33(1):159-74. DOI: http://dx.doi.org/10.2307/2529310

24. O'Cathain A, Brazier JE, Milner PC, Fall M. Cost effectiveness of minor surgery in general practice: a prospective comparison with hospital practice. Br J Gen Pract. 1992;42(354):13-7.

25. McWilliam LJ, Knox F, Wilkinson N, Oogarah P. Performance of skin biopsies by general practitioners. BMJ. 1991;303(6811):1177-9. DOI: http://dx.doi.org/10.1136/bmj.303.6811.1177

26. Vaquero Martínez JJ, García Aparicio JM, Díaz Gómez J, Blasco Paredes D. Efficiency of minor surgery in primary care according to the costs. Aten Primaria. 2002;30(2):86-91.

27. Wickstrom GC, Kolar MM, Keyserling TC, Kelley DK, Xie SX, Bognar BA, et al. Confidence of graduating internal medicine residents to perform ambulatory procedures. J Gen Intern Med. 2000;15(6):361-5. DOI: http://dx.doi.org/10.1046/j.15251497.2000.04118.x

28. Sempowski IP, Rungi AA, Seguin R. A cross sectional survey of urban Canadian family physicians' provision of minor office procedures. BMC Fam Pract. 2006;7:18. DOI: http://dx.doi.org/10.1186/1471-2296-7-18

29. Gmajnić R, Pribić S, Lukić A, Ebling B, Cupić N, Marković l. Effect of surgical training course on performance of minor surgical procedures in family medicine physicians' offices: an observational study. Croat Med J. 2008;49(3):358-63. DOI: http://dx.doi.org/10.3325/cmj.2008.3.358 
${ }^{\text {a }}$ Residência de Medicina de Família e Comunidade do Rio de Janeiro (Clínica da Família Zilda Arns). Rio de Janeiro, RJ, Brasil. diaslacerda@gmail.com (Autor correspondente)

b Universidade Estácio de Sá/RJ (UNESA/RJ). Rio de Janeiro, RJ, Brasil. cassiobserafini@gmail.com; leoj86@ hotmail.com

c Universidade Federal do Rio de Janeiro (UFRJ). Rio de Janeiro, RJ, Brasil. pedromivianna@gmail.com; giulia.regattieri@hotmail.com 